\title{
Public Health Informatics and the H1N1 Pandemic
}

\author{
Joseph Lombardo, Johns Hopkins University Applied Physics Laboratory
}

On June 11th 2009, Dr. Margaret Chan, Director-General of the World Health Organization (WHO), announced that the Influenza alert level was being raised from a Phase 5 to Phase 6 indicating the start of the 2009 Pandemic. The pandemic resulted from a previously uncirculated strain of H1N1 Influenza which spread rapidly in the spring of 2009. The second wave of the pandemic began as schools reopened in the late summer. At the time of the writing of this commentary, H1N1 was widely spread in 48 states and Canada. Due to the intensive surveillance and preparedness activities surrounding a rapidly spreading avian H5N1 influenza, the WHO and the Centers for Disease Control and Prevention, were able to closely monitor the progression of the H1N1 strain in the human population and initiate an accelerated vaccination program. This heightened activity comes at a time of reduced budgets for state and local health departments who have primary responsibility for monitoring and containment within their jurisdictions.

As resources become more limited, public health workers are forced to focus their attention on the most urgent priorities. In order to effectively multi-task, public health must leverage new technologies to get more done with existing resources. Public health informatics is an emerging field that has the potential to immediately support the ten essential public health services. Automated case specific disease monitoring applications can now collect, analyze and present to the user information that would have taken days to assemble only a few years ago. Applications such as ESSENCE, RODS, and BioSense have been able to monitor spread of $\mathrm{H} 1 \mathrm{~N} 1$ at state, local, and national levels. The Internet has become an educational tool for informing the population on the latest research to maintain and improve health. The websites of local health departments, CDC, WHO, WebMD, Google and many others have all seen increased activity as primary caregivers seek information on how to protect their family members from H1N1. Emerging knowledge repositories showcased at national conferences have demonstrated the ability to transparently provide public health alerts to clinicians during patient encounters. Information is provided to support disease management while enhancing real-time monitoring. The Internet provides a secure collaborative environment to support public health monitoring across jurisdictional boundaries. The DiSTRIBuTE project creates a national view of H1N1 trends from data provided by public health agencies across the country that have the desire to collaborate with their counterparts in other jurisdictions. These are just a few public health informatics applications that support H1N1 containment.

As the field of public health informatics expands, its researchers and developers must keep in mind the goal of translating their achievements into open environment so that their informatics products can be made available to public health practitioners when they are needed. Traditionally, informatics tools are presented to the community at conferences and through journal articles. Local public health funding limitations may restrict travel at conferences or membership in societies that publish articles in their journals. The Online Journal of Public Health Informatics (OJPHI) provides an open access vehicle that supports the presentation of the latest open source informatics tools to the public health community to support their translation to practice. As an open access communications vehicle to public health, articles published in OJPHI could provide knowledge of open source informatics tools to help public health manage the next emerging health risk. 